\title{
Treatment of lymphatic leakage after retroperitoneal tumor resection by lymphangiography and embolization: A case report
}

\author{
JIAWEI GAO, YONG JIN and WEI CHEN \\ Department of General Surgery, Second Affiliated Hospital of Soochow University, \\ Suzhou, Jiangsu 215004, P.R. China
}

Received December 18, 2019; Accepted June 25, 2020

DOI: 10.3892/ol.2020.11916

\begin{abstract}
Lymphatic leakage is an infrequent but relatively serious complication after resection of retroperitoneal tumors. Although different approaches have been attempted in treatment of lymphatic leakage, none of them has been consistently effective. This study report on a female patient, aged 55-years with massive retroperitoneal tumor, with lymphangiography and embolization for resolution of lymphatic leakage after retroperitoneal tumor resection. Computed tomography revealed retroperitoneal cystadenoma. After the right ureteral stent was placed, the retroperitoneal tumor resection was performed, and after operation, the patient presented with lymphatic leakage unresponsive to several treatment measures. From the thirtyseventh postoperative day (POD37), the patient underwent lymphangiography and embolization, which resolved her lymphatic leakage. Lymphangiography is useful for detecting lymphatic leakage occurring after retroperitoneal tumor resection. Furthermore, lymphatic embolization is feasible, effective, and safe for managing leaks demonstrated on lymphangiography.
\end{abstract}

\section{Introduction}

The work is reported in line with the SCARE criteria (1). The treatment of retroperitoneal tumor is frequently surgical resection and lymph node dissection, which usually followed by lymphatic leakage. A variety of approaches have been attempted to the treatment of lymphatic leakage. However, so far none has been consistently effective or optimal (2). This study report a case of lymphatic leakage after retroperitoneal

Correspondence to: Dr Wei Chen, Department of General Surgery, Second Affiliated Hospital of Soochow University, 1055 Sanxiang Road, Suzhou, Jiangsu 215004, P.R. China

E-mail: e826ab@163.com

Key words: lymphatic leakage, retroperitoneal tumor, treatment, lymphangiography, embolization tumor resection that was successfully resolved by lymphangiography and embolization.

\section{Case report}

The study was approved by the Ethics Committee of the Second Affiliated Hospital of Soochow University (Suzhou, China). The patient who participated in this research provided a signed informed consent and had complete clinical data. The patient was a 55-year-old female patient that had abdominal mass and complained that the mass gradually increased to affect sleep. B-ultrasound examination revealed mixed echo zone with approximately $243 \times 118 \mathrm{~mm}$ in size in the abdominal cavity. Computed tomography (CT) showed a large mass on the right side of the abdomen, with the right ureter and inferior vena cava compressed to the right, indicating retroperitoneal cystadenoma. Magnetic resonance imaging (MRI) suggested a large multilocular cystic space in the retroperitoneal and hepatorenal space, approximately $261 \times 181 \times 150 \mathrm{~mm}$ in size, which was very likely to be epidermoid cyst (Fig. 1). After admission, physical examination showed a hard, local uplift in the right abdomen, about $25 \times 12 \mathrm{~cm}$ in size, without tenderness, fixation or any other positive signs. After placing the right ureteral stent, the retroperitoneal tumor resection was performed. As a result, intraoperative exploration revealed a large cystic solid tumor in the right abdominal cavity, which was multilocular and lobulated. The inferior vena cava and ureter were pushed up to the right abdominal wall and the right kidney was moved up to the lower part of the liver. After cautious separation along the tumor to protect the blood vessels and ureters, careful irrigation was performed before the peritoneum was closed. No active bleeding or obvious lymphatic leakage was detected. Pelvic cavity was then placed, followed by placing two drainage tubes at the incision.

Postoperative pathology: (Posterior peritoneal) mature cystic teratoma showed mucinous tumor and mild to moderate atypical hyperplasia of glandular epithelium. After the operation, the abdominal drainage tube had a small amount in light liquid of blood. CT revealed peritoneal effusion after operation on POD7 (Fig. 2), therefore, peritoneocentesis was performed, and $200 \mathrm{ml}$ of yellow-white, turbid liquid was extracted. Two abdominal drainage tubes were removed on the same day. After two days of observation, there was 


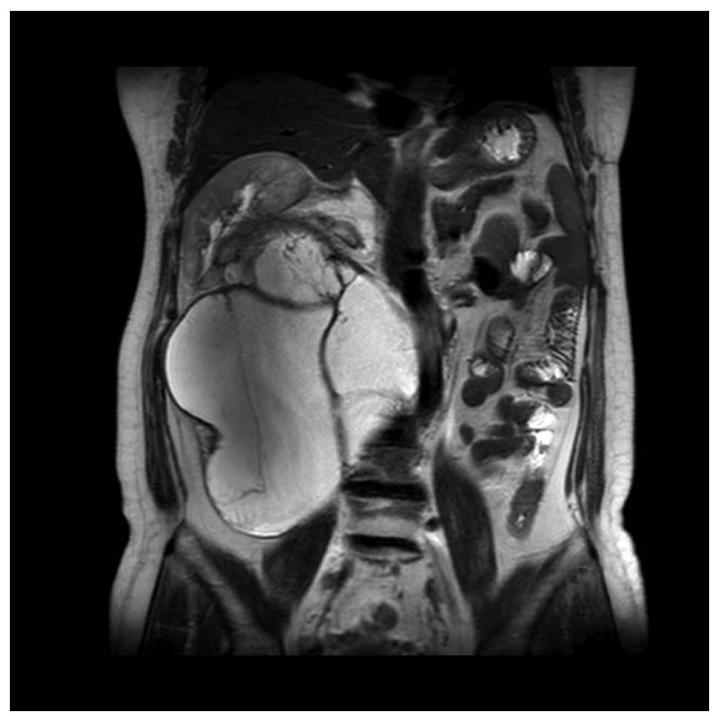

Figure 1. Coronal MRI of the abdomen shows a large retroperitoneal tumor. The organs have deviated from their normal position. MRI, magnetic resonance imaging.

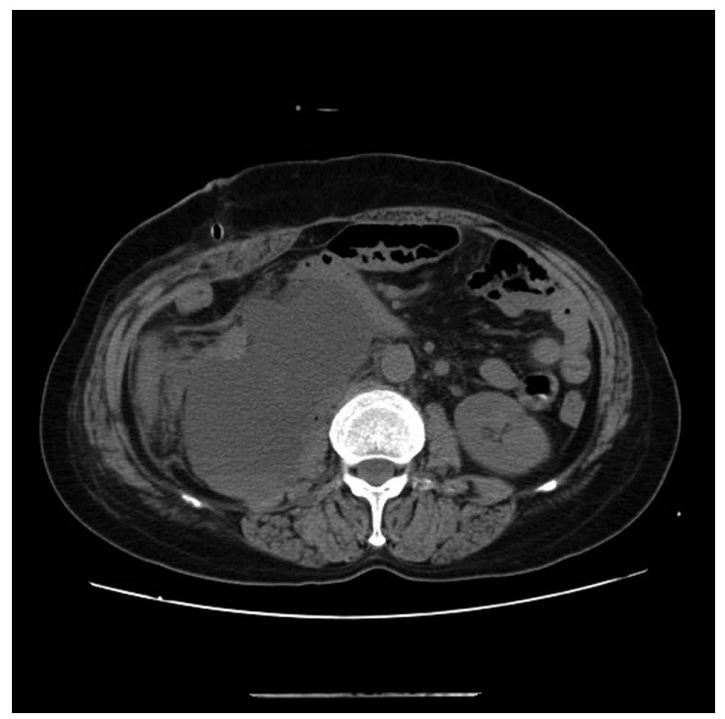

Figure 2. Abdominal CT scan shows localized retroperitoneal lymphatic leakage. CT, computed tomography.

no decrease in the amount of abdominal drainage fluid. The chylous qualitative test of concurrent drainage fluid was performed, with positive Sudan staining. Therefore, the patient was instructed to eat high-calorie, high-protein, low-fat fluid, in order to improve parenteral nutrition, and keep the drainage tube unobstructed. In addition, electrolyte was regularly reviewed to prevent water and electrolyte balance disorder, and the daily drainage volume was maintained between 700-1,100 $\mathrm{ml}$ thereafter. Subsequently, in the first month after operation, lymph node lipiodolography and embolization were performed under ultrasound guidance. Meilan $(1 \mathrm{ml})$ was injected between the toes of the patient, after $15 \mathrm{~min}$ the lymphatic vessels on the instep were clearly blue. Target lymphatic vessels were then selected for local anesthesia, skin incision and lymphatic vessel separation. Then lymphatic vessels were punctured and fixed.

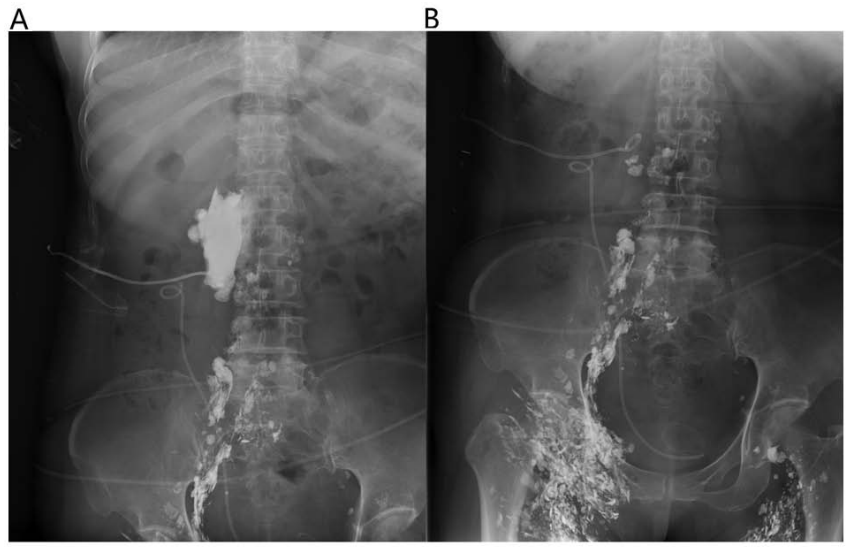

Figure 3. Lymphangiography shows massive exudation of lymph, the leakage was reduced on POD37 compared with POD30, but it was not completely occluded. (A) First lymphography; (B) second lymphography.

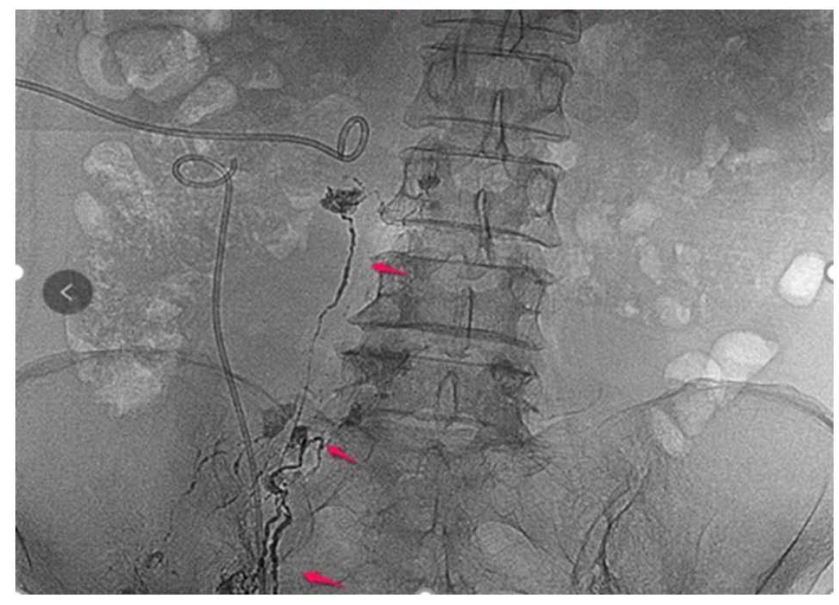

Figure 4 . The arrows point to the thin strips of lymphatic vessels and leakage development.

Lymphangiography was performed with a 2:1 mixture of iodide oil (Guerbet) and NBCA puncture embolization $(3,4)$. Daily drainage was decreased after lipiodolography, and not obvious. One week later, the lymph node lipiodolography and embolization were re-performed (Fig. 3). As shown, the leakage of the exudation site was reduced. One day after the lipiodolography, $125 \mathrm{ml}$ of milky liquid was drained, which suggested obviously covered leakage area and relatively limited scope.

Afterwards, the patient was instructed to fast and switch to total parenteral nutrition for one week. The drainage tube was clamped and the patient did not complain of any discomfort. After two weeks of lipiodolography, the peritoneal puncture drainage tube was removed, and the patient was instructed to eat a light diet and gradually make a transition. After one week of observation, no peritoneal effusion was found by b-ultrasound and the patient was discharged from hospital. So far, the patient has been reviewed every three months for one and a half years without any discomfort. After performing lymphangiography and embolization, there was no series of postoperative complications such as edema or skin thickening in the lower extremities. 


\section{Discussion}

Lymphatic leakage is a well-known complication following retroperitoneal tumor resection (5). Many attempts have been made in the treatment of lymphatic leakage, as listed: i) Abdominal drainage: In spite of no direct evidence supporting that abdominal drainage can promote the healing of fistula, it can provide clinical diagnostic basis and alleviate a series of clinical symptoms such as abdominal pain and distension to a certain extent. In addition, the therapeutic treatment plan can be adjusted according to the amount of drainage fluid. ii) Antisecosis: Mid-chain triglyceride diet with parenteral nutrition can reduce the amount of lymphatic leakage, which is because the short-chain triacylglycerol contained in food can be absorbed directly into the blood through the intestinal tract, while the long-chain triacylglycerol should be transported and absorbed through the lymphatic pathway (6). iii) Octreotide and somatostatin: It has been reported that the addition of octreotide in food exert a significantly early scavenging effect on postoperative lymphatic drainage of patients, mainly to prevent the conversion of triglycerides in the diet into free fatty acids in the intestinal tract, thereby reducing the absorption of fatty acids $(7,8)$. In recent years, surgical intervention has been reported to be guided by near-infrared fluorescence imaging technology, and indocyanine green can be used to locate leakage hot spots, providing high sensitivity and real-time imaging to help surgeons perform preventive ligation in cases where it is needed. This technique may have the potential to more accurately diagnose and treat lymphatic leakage during surgery (9).

Nevertheless, some leaks still persist despite conservative treatment, therefore, more effective treatments are needed (10). Currently, lymphangiography in conjunction with embolization is a relatively safe, powerful, and reliable interventional method (11).

Lymphatic intervention is less invasive compared with surgery, involving injection of ethiodized oil into the lymphatic system to obtain a lymphangiogram (12). In addition to its diagnostic value, lymphangiography has also been reported to have therapeutic effects (11). This is possibly due to the high viscosity of contrast medium such as ethiodized oil, which can stimulate the growth of local new granulation tissue, triggering a series of inflammatory reactions to decrease leakage. In this case report, two procedures of lymphangiography and embolization were performed, in which thin strips of lymphatic vessels and leakage development were observed, however, without immediate effect (Fig. 4). The second attempt was successful and the amount of drainage was decreased from a maximum output of 700 to $125 \mathrm{ml} /$ day after lymphangiography.

This case demonstrates postoperative lymphatic leakage that was successfully treated by performing repeated lymphangiography and embolization. This technique should be considered for further application and study of lymphatic leakage after abdominal surgery and lymphadenectomy. However, this case has certain limitations. First, the patient is followed up for a short period of time. Second, there is a lack of multi-sample retrospective analysis to compare the effects of different treatment methods on the prognosis of patients with postoperative lymphatic leakage. Nowadays, intranodal lymphangiography and ultrasound-guided lymphangiography are also used in clinical practice, resulting in less trauma to patients and better positioning accuracy, which can be further studied (11).

\section{Acknowledgements}

Not applicable.

\section{Funding}

No funding was received.

\section{Availability of data and materials}

The datasets used and/or analyzed during the current study are available from the corresponding author on reasonable request.

\section{Authors' contributions}

JG wrote the manuscript, interpreted and analyzed the data. WC designed the study and performed the experiments. YJ was responsible for the analysis and discussion of the data. All authors read and approved the final manuscript.

\section{Ethics approval and consent to participate}

The study was approved by the Ethics Committee of the Second Affiliated Hospital of Soochow University (Suzhou, China). The patient who participated in this research provided a signed informed consent and had complete clinical data.

\section{Patient consent for publication}

Written informed consent was obtained from the patient for the publication of this case report and any accompanying images.

\section{Competing interests}

The authors declare that they have no competing interests.

\section{References}

1. Agha RA, Borrelli MR, Farwana R, Koshy K, Fowler AJ and Orgill DP; SCARE Group: The SCARE 2018 Statement: Updating consensus Surgical CAse REport (SCARE) guidelines. Int J Surg 60: 132-136, 2018.

2. Kim EA, Park H, Jeong SG, Lee C, Lee JM and Park CT: Octreotide therapy for the management of refractory chylous ascites after a staging operation for endometrial adenocarcinoma. J Obstet Gynaecol Res 40: 622-626, 2014.

3. Nadolski GJ, Chauhan NR and Itkin M: Lymphangiography and lymphatic embolization for the treatment of refractory chylous ascites. Cardiovasc Intervent Radiol 41: 415-423, 2018.

4. Inoue M,Nakatsuka S, Yashiro H, Tamura M, Suyama Y, Tsukada J, Ito N, Oguro S and Jinzaki M: Lymphatic intervention for various types of lymphorrhea: Access and treatment. Radiographics 36: 2199-2211,2016.

5. Capocasale E, Iaria M, Vistoli F, Signori S, Mazzoni MP, Dalla Valle R, De Lio N, Perrone V, Amorese G, Mosca F, et al: Incidence, diagnosis, and treatment of chylous leakage after laparoscopic live donor nephrectomy. Transplantation 93: 82-86, 2012.

6. Steven BR and Carey S: Nutritional management in patients with chyle leakage: A systematic review. Eur J Clin Nutr 69: 776-780, 2015. 
7. Weniger M, D'Haese JG, Angele MK, Kleespies A, Werner J and Hartwig W: Treatment options for chylous ascites after major abdominal surgery: A systematic review. Am J Surg 211: 206-213, 2016

8. Seow C, Murray L and McKee RF: Surgical pathology is a predictor of outcome in post-operative lymph leakage. Int $\mathbf{J}$ Surg 8: 636-638, 2010.

9. Yang F, Zhou J, Li H, Yang F, Xiao R, Chi C, Tian J and Wang J: Near-infrared fluorescence-guided thoracoscopic surgical intervention for postoperative chylothorax. Interact Cardiovasc Thorac Surg 26: 171-175, 2018.

10. Kortes N, Radeleff B, Sommer CM, Bellemann N, Ott K, Richter GM, Kauczor HU and Stampfl U: Therapeutic lymphangiography and CT-guided sclerotherapy for the treatment of refractory lymphatic leakage. J Vasc Interv Radiol 25: 127-132, 2014.
11. Lee EW, Shin JH, Ko HK, Park J, Kim SH and Sung KB: Lymphangiography to treat postoperative lymphatic leakage: A technical review. Korean J Radiol 15: 724-732, 2014.

12. Iwai T, Uchida J, Matsuoka Y, Kosoku A, Shimada H, Nishide S, Kabei K, Kuwabara N, Yamamoto A, Naganuma T, et al: Experience of lymphangiography as a therapeutic tool for lymphatic leakage after kidney transplantation. Transplant Proc 50: 2526-2530, 2018. 